\title{
The impact of the Catechol-O-methyltransferase Val I58Met polymorphism on survival in the general population - the HUNT study
}

Knut Hagen*1,2, Lars J Stovner ${ }^{1,2}$, Frank Skorpen ${ }^{3}$, Elin Pettersen ${ }^{3}$ and JohnAnker Zwart ${ }^{1,2,4}$

\begin{abstract}
Address: ${ }^{1}$ Department of Clinical Neuroscience, Faculty of medicine, Norwegian University of Science and Technology, Trondheim, Norway, ${ }^{2}$ Norwegian National Headache Centre, Section of Neurology, St. Olavs Hospital, Trondheim, Norway, ${ }^{3}$ Department of Laboratory Medicine, Children's and Women's health, Faculty of medicine, Norwegian University of Science and Technology, Trondheim, Norway and ${ }^{4}$ National Centre for Spinal Disorders, St. Olavs Hospital, Trondheim, Norway

Email: Knut Hagen* - knut.hagen@ntnu.no; Lars J Stovner - lars.stovner@ntnu.no; Frank Skorpen - frank.skorpen@ntnu.no; Elin Pettersen - elin.pettersen@ntnu.no; John-Anker Zwart - john-anker.zwart@ntnu.no

* Corresponding author
\end{abstract}

Published: 19 June 2007

BMC Medical Genetics 2007, 8:34 doi:10.1 |86//47|-2350-8-34

This article is available from: http://www.biomedcentral.com/I47I-2350/8/34

(C) 2007 Hagen et al; licensee BioMed Central Ltd.

This is an Open Access article distributed under the terms of the Creative Commons Attribution License (http://creativecommons.org/licenses/by/2.0), which permits unrestricted use, distribution, and reproduction in any medium, provided the original work is properly cited.
Received: 20 October 2006

Accepted: 19 June 2007

\begin{abstract}
Background: The catechol-O-methyltransferase (COMT) gene contains a functional polymorphism, Vall58Met which has been related to common diseases like cancer, psychiatric illness and myocardial infarction. Whether the Val I58Met polymorphism is associated with survival has not been evaluated in the general population. The aim of this prospective study was to evaluate the impact of codon 158 COMT gene polymorphism on survival in a population-based cohort.
\end{abstract}

Methods: The sample comprised 2979 non-diabetic individuals who participated in the NordTrøndelag Health Study (HUNT) in the period 1995-97. The subjects were followed up with respect to mortality throughout year 2004.

Results: 212 men and 183 women died during the follow up. No association between codon 158 COMT gene polymorphism and survival was found. The unadjusted relative risk of death by nonischemic heart diseases with Met/Met or Met/Val genotypes was 3.27 ( $95 \%$ confidence interval, I. 19-9.00) compared to $\mathrm{Val} / \mathrm{Val}$ genotype. When we adjusted for age, gender, smoking, coffee intake and body mass index the relative risk decreased to 2.89 (95\% confidence interval, I.04-8.00).

Conclusion: During 10 year of follow-up, the Val I58Met polymorphism had no impact on survival in a general population. Difference in mortality rates from non-ischemic heart diseases may be incidental and should be evaluated in other studies.

\section{Background}

The catechol-O-methyltransferase (COMT) gene located at chromosome 22q11.2 contains a functional polymorphism at codon 158 that has been the subject of several molecular epidemiological studies because of the impor- tant role of the COMT enzyme in the metabolism of catecholamines and catechol estrogens. A substitution of valine (Val) by methionine (Met) at codon 158 affects the activity of the COMT enzyme, and individuals with the Val/Val genotype have a three- to fourfold higher activity 
of the COMT enzyme than those with Met/Met genotype [1].

The Val158Met polymorphism has been linked to e.g. psychiatric disorders and pain perception [2-4], and several epidemiological studies have also reported an association to several potential fatal disorders. Presence of the $\mathrm{Val} / \mathrm{Val}$ genotype has been considered to be favorable because it seems to lower the risk of developing nonHodgkin lymphoma and estrogen-associated cancers in women [5-7], and because it is associated with a higher tendency to remain free from increase in prostate-specific antigen in men with prostate cancer [8]. Men with Val/Val genotype may also be less likely to commit suicide [9], and the Val allele has proved to be associated with less aggressive behaviors or violent suicide attempts in schizophrenic patients $[10,11]$. On the other hand, the Val/Val genotype seems to give a higher risk of myocardial infarction among hypertensive patients [12], and it has also been associated with high systolic blood pressure [13].

Despite the large number of studies evaluating the relationship between Val158Met polymorphism and diseases, the influence of this polymorphism on survival has not been evaluated. The aim of this prospective study was to evaluate the impact of codon 158 COMT gene polymorphism on survival in a population-based cohort.

\section{Results}

The genotype distribution among the 2979 individuals (1347 men and 1632 women) was in Hardy-Weinberg equilibrium. The demographic data of the different genotype groups are shown in Table 2 . The individuals in the three genotype groups did not differ significantly with regard to gender, age, body mass index, cholesterol level, smoking status, level of physical activity, coffee intake,
Table I: Primers and hybridization probes used for COMT Vall58Met genotyping

\begin{tabular}{ll}
\hline & Sequence \\
\hline Primers: & Forward 5'-ACGCCGTGATTCAGGAGCA-3' \\
& Reverse 5'-GTCTTTCCTCAGCCCCAG-3' \\
Probes: & Sensor 5'-TCACGCCAGCGAAATCCA-FI*-3' \\
& Anchor 5' LC Red 640\#-ATCCGCTGGGTGATGGCG-3'
\end{tabular}

$* \mathrm{FI}=$ Fluorescein

\# LC Red 640 = Light Cycler Red 640

Underlined $\mathrm{C}$ indicates polymorphic site

prevalence of stroke, or education level. However, the prevalence of ischemic heart diseases (including angina pectoris and/or myocardial infarction) reported in connection with the HUNT study in 1995-97 tended to be lower among individuals with $\mathrm{Val} / \mathrm{Val}$ genotype than among those with other genotypes (6.4 percent vs. 8.6 percent, $p=0.09$ ). The individuals with known genotype were significantly older and had, accordingly, higher mean systolic and diastolic BP and higher mean cholesterol level than those without COMT data available $(p<$ 0.05) (Table 2).

\section{Overall mortality}

212 (15.7 percent) men and 183 (11.2 percent) women died during the follow up $(p<0.001)$. The distribution of the COMT Val158Met alleles was similar among survivors and the dead (Table 3), evident for both genders (data not shown). No difference in survival was found for the different genotypes (Fig. 1).

\section{Death caused by neoplasms (ICD-I0 C00-D48)}

61 men (4.5 percent) and 44 women (2.7 percent) ( $p=$ $0.007)$ had neoplasm as the underlying cause of death. No difference in cancer mortality was found between the dif-

Table 2: Clinical characteristics of the subjects according to COMT genotypes.

\begin{tabular}{|c|c|c|c|c|}
\hline Characteristics & No COMT genotyping $(n=62,247)$ & Met/Met $(n=95 I)$ & Met/Val $(n=1482)$ & Val/Val $(n=546)$ \\
\hline Sex, female (\%) & 53.2 & 54.0 & 55.1 & 55.1 \\
\hline Age, mean (SD) & $49.1(18.9)^{\$}$ & $52.8(18.3)$ & $52.7(18.2)$ & $52.1(18.0)$ \\
\hline Education $>12$ years $(\%)$ & 19.0 & 17.4 & 15.7 & 16.7 \\
\hline Mean systolic blood pressure $(\mathrm{mmHg})(\mathrm{SD})$ & $137.6(22.3)^{\$}$ & $140.9(22.2)$ & $140.4(22.6)$ & $141.6(22.9)$ \\
\hline Mean diastolic blood pressure $(\mathrm{mmHg})(\mathrm{SD})$ & $80.2(12.5)^{\$}$ & $81.7(12.4)$ & $81.0(12.6)$ & $82.3(12.9)$ \\
\hline Body mass index, $\mathrm{kg} / \mathrm{m}^{2}(\mathrm{SD})$ & $26.4(4.1)$ & $26.3(4.2)$ & $26.4(4.1)$ & $26.3(4.2)$ \\
\hline Cholesterol, mmol (SD) & $5.89(1.26)^{\$}$ & $6.02(1.31)$ & $6.03(1.30)$ & $6.08(1.37)$ \\
\hline Current smoking (\%) & 29.0 & 28.0 & 29.6 & 29.0 \\
\hline High level of physical activity* (\%) & 24.1 & 19.9 & 18.7 & 20.1 \\
\hline Coffee intake $>6$ cups/day $(\%)$ & 20.1 & 19.6 & 18.6 & 19.0 \\
\hline Use of antihypertensive medication (\%) & 11.0 & 12.6 & 13.3 & 11.2 \\
\hline Myocardial infarction (\%) & 3.3 & 3.7 & 3.5 & 2.8 \\
\hline Angina pectoris (\%) & 5.0 & 6.9 & 7.1 & 5.9 \\
\hline Stroke (\%) & 1.9 & 2.5 & 1.6 & 1.8 \\
\hline
\end{tabular}

* High level of physical activity was defined as high intensity exercise more than I hour per week.

$\$ p<0.05$ 
Table 3: Mortality from various causes by January I, 2005 related to COMT genotypes and 5- and I0-year survival.

\begin{tabular}{|c|c|c|c|c|c|c|c|c|}
\hline & \multicolumn{2}{|c|}{ Met $/$ Met $(n=95 I)$} & \multicolumn{2}{|c|}{ Met/Val $(n=1482)$} & \multicolumn{2}{|c|}{$\mathrm{Val} / \mathrm{Val}(n=546)$} & \multirow{2}{*}{$\begin{array}{c}\text { 5-year survival } \\
\mathrm{P}^{*}\end{array}$} & \multirow{2}{*}{$\begin{array}{c}\text { I0-year survival } \\
\mathrm{p}^{*}\end{array}$} \\
\hline & $\mathrm{n}$ & $\%$ & $\mathrm{n}$ & $\%$ & $\mathrm{n}$ & $\%$ & & \\
\hline \multicolumn{9}{|l|}{ Genotypes } \\
\hline Total deaths & 129 & 32.7 & 197 & 49.8 & 69 & 17.5 & 0.77 & 0.87 \\
\hline Neoplasms (C00-D48) & 35 & 33.3 & 52 & 49.5 & 18 & I7.I & 0.97 & 0.93 \\
\hline Cardiovascular causes (100-199) & 58 & 31.9 & 92 & 50.5 & 32 & 17.6 & 0.47 & 0.96 \\
\hline All other causes & 36 & 33.3 & 53 & 49.1 & 19 & 17.6 & 0.72 & 0.94 \\
\hline Alleles & Met & & Val & & & & & \\
\hline Total deaths & 455 & 57.6 & 335 & 42.4 & & & 0.35 & 0.63 \\
\hline Neoplasms (C00-D48) & 122 & 58.1 & 88 & 41.9 & & & 0.74 & 0.48 \\
\hline Cardiovascular causes (100-199) & 208 & 57.1 & 156 & 42.9 & & & 0.30 & 0.57 \\
\hline All other causes & 125 & 57.9 & 91 & 42.1 & & & 0.98 & 0.51 \\
\hline
\end{tabular}

* $\mathrm{p}$ value for the log-rank test

ferent Val158Met genotype groups (Table 4). Cancer in the digestive system (C00-C26) was common among women (39 percent), whereas 20 men (33 percent) died because of prostate cancer (C61). Slightly more men with $\mathrm{Val} /$ Val genotype had prostate cancer as underlying cause of death than those with other genotypes (2.0 percent versus 1.4 percent, $p=0.44$, long-rank test). However, no such tendency was found when including 8 men with prostate cancer as immediate or contributing cause of death (2.0 percent versus 2.0 percent, $p=0.95$, log-rank test)

\section{Cardiovascular causes (ICD-10 100-100)}

Cardiovascular disease was the underlying cause of death in 97 men (7.2 percent) and 85 women (5.2 percent) ( $p=$

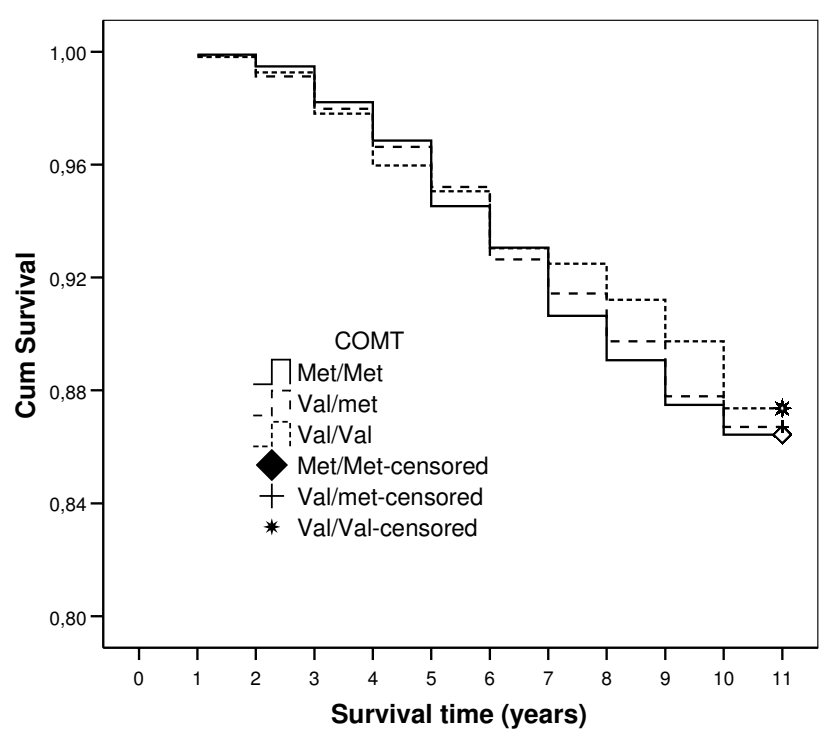

Figure I

Survival curves for different genotypes. Log-rank test: $p=$ 0.87 .
0.02). Among these, 87 individuals died of ischemic heart disease (I20-I25) and 34 of non-ischemic heart diseases (I30-I33 or I39-I52) (Table 4). When the Met/Met and $\mathrm{Val} / \mathrm{Met}$ genotypes were pooled, a borderline significant tendency to lower mortality caused by non-ischemic heart diseases was found among individuals with $\mathrm{Val} / \mathrm{Val}$ genotype $(\mathrm{n}=2)$ compared to those with other genotypes $(\mathrm{n}=$ 32) ( $p=0.06$, rank-log test). Among the 62 individuals with non-ischemic heart diseases as underlying $(\mathrm{n}=34)$ or immediate cause $(\mathrm{n}=28)$ of death, subjects with $\mathrm{Val} / \mathrm{Val}$ had lower mortality rates $(\mathrm{n}=4)$ than those with the Met/ Val or Met/Met genotypes $(\mathrm{n}=58)(p=0.015$, rank-log test) (Figure 2). The unadjusted relative risk of death by non-ischemic heart diseases with Met/Met or Met/Val genotypes was 3.27 (95\% confidence interval, 1.19-9.00) compared to $\mathrm{Val} / \mathrm{Val}$ genotype. When we adjusted for age, gender, smoking, coffee intake and body mass index the relative risk decreased to 2.89 (95\% confidence interval, 1.04-8.00).

\section{Mortality among 69 with self-reported diabetes mellitus}

During the 10 year follow up the overall mortality was more than three times higher among the excluded 69 individuals with self-reported diabetes mellitus than among the included sample of 2979 non-diabetic subjects $(47.8 \%$ versus $13.3 \%, \mathrm{p}<0.001)$. However, our results were not substantially changed when we repeated the analyses including these 69 diabetic persons. Specifically, out of the 33 diabetic who died during the follow up, a total of 6 had non-ischemic heart diseases as underlying ( $\mathrm{n}$ $=3)$ or immediate cause $(\mathrm{n}=3)$ of death. Even when these were included, subjects with $\mathrm{Val} / \mathrm{Val}$ had lower mortality rates $(n=6)$ than those with the Met/Val or Met/Met genotypes $(n=62)(p=0.04$, rank-log test $)$.

\section{Discussion}

In this population-based sample of individuals the Val158Met polymorphism at the COMT gene had no impact on survival in general. 
Table 4: Causes of death according to COMT genotypes

\begin{tabular}{|c|c|c|c|c|c|c|c|}
\hline & \multicolumn{2}{|c|}{ Met/Met $(n=95 I)$} & \multicolumn{2}{|c|}{ Met/Val $(n=1482)$} & \multicolumn{2}{|c|}{$\mathrm{Val} / \mathrm{Val}(n=546)$} & \multirow{2}{*}{$\begin{array}{c}\text { Val/Val vs. other genotypes } \\
\text { Log-rank p value }\end{array}$} \\
\hline & $\mathrm{n}$ & $\%$ & $\mathrm{n}$ & $\%$ & $\mathrm{n}$ & $\%$ & \\
\hline Neoplasms (C00-D48) & 35 & 3.7 & 52 & 3.5 & 18 & 3.3 & 0.74 \\
\hline Cancers in the digestive system (C00-C26) & 14 & 1.5 & 14 & 0.9 & 4 & 0.7 & 0.39 \\
\hline Cancer in the respiratory system $(\mathrm{C} 30-\mathrm{C} 39)$ & 6 & 0.6 & $\mathrm{II}$ & 0.7 & 1 & 0.2 & 0.23 \\
\hline Breast cancer/cancer in female genital organs (C50-C58) & 2 & 0.2 & 2 & 0.1 & I & 0.2 & 1.00 \\
\hline Prostate cancer $(\mathrm{C} 6 \mathrm{I})$ & 5 & 0.5 & 10 & 0.7 & 6 & I.I & 0.24 \\
\hline All other neoplasms & 8 & 0.8 & 15 & 1.0 & 6 & I.I & 0.92 \\
\hline Cardiovascular causes (100-199) & 58 & 6.1 & 92 & 6.2 & 32 & 5.9 & 0.77 \\
\hline Ischemic heart diseases (120-125) & 29 & 3.0 & 43 & 2.9 & 15 & 2.7 & 0.78 \\
\hline Non-ischemic heart diseases (130-133 and I39-152)\# & 12 & 1.3 & 20 & 1.3 & 2 & 0.4 & 0.06 \\
\hline Cerebrovascular diseases (160-169) & 10 & I.I & 19 & 1.3 & 9 & 1.6 & 0.39 \\
\hline All other vascular deaths & 7 & 0.7 & 10 & 0.7 & 6 & I.I & 0.33 \\
\hline Sudden death (R96) & I & 0.1 & 3 & 0.2 & I & 0.2 & 0.92 \\
\hline Accidents (V0I-V99, W00-W99, and X00-X99) & 7 & 0.7 & 10 & 0.7 & 5 & 0.9 & 0.87 \\
\hline Dementia (F03, G30, and R54) & 7 & 0.7 & 6 & 0.4 & 1 & 0.2 & 0.49 \\
\hline Diseases of the respiratory system (J00-J99) & 12 & 1.3 & 13 & 0.9 & 5 & 0.9 & 0.95 \\
\hline Diseases of the digestive system (KO0-K93) & 3 & 0.3 & 7 & 0.5 & 4 & 0.7 & 0.33 \\
\hline Diseases of the kidney and ureter (N00-99) & 3 & 0.3 & 5 & 0.3 & 0 & 0 & 0.31 \\
\hline Other diseases* & 3 & 0.3 & 9 & 0.6 & 3 & 0.5 & 0.87 \\
\hline
\end{tabular}

\# Non-ischemic heart diseases (130-133 and 139-152): Underlying cause of death among 34 individuals. Additionally, immediate cause of death among 28 others (in total 62 individuals)

* Infections (A00-B99), diseases of the nervous system (G00-G99), mental and behavioral disorders (F00-F99), and other symptoms, sign, abnormal findings, ill-defined causes (R00-R94).

The strength of this study was the prospective cohort design which provides a valuable complement to casecontrol studies and with ability to adjust for potential confounding factors [19]. Furthermore, the COMT genotyping was performed in a random sample of individuals

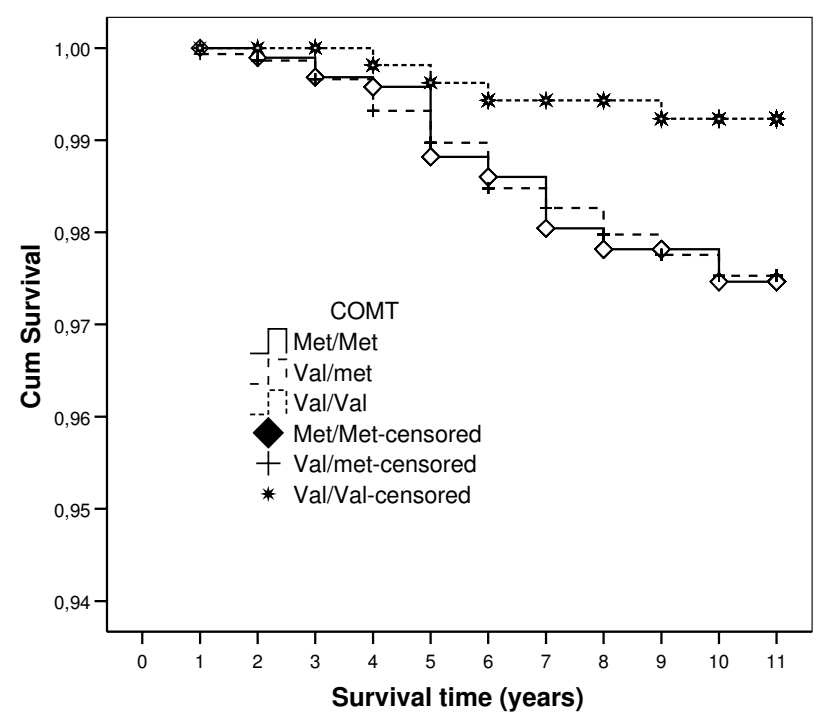

Figure 2

Survival curves for different genotypes considering only death from non-ischemic heart diseases (130-133, 139-152). Log-rank test for $\mathrm{Val} / \mathrm{Val}$ versus other genotypes: $p=0.015$. drawn from a genetically homogenous white Norwegian population and being unselected except from the fact that they did not have diabetes. Less than 3 percent of subjects were of non-white ethnicity. Bias caused by admixed ethnicities may be reduced in population-based studies when a genetically homogenous population is investigated.

The majority of included participants were selected completely at random, but were mixed together with a nondiabetic group that were age-matched controls for the purpose of a different genetic study on diabetes. It would have been preferable to perform the analyses on a completely random selected population, including both diabetic and non-diabetic persons, but this strategy was not possible because of the format of the data file. Because diabetes status was a selection criterion for approximately 30 percent of the sample, we decided to exclude 69 individuals with diabetes mellitus, making the whole group more homogenous. Individuals with $\mathrm{Val} / \mathrm{Val}$ seem to be less likely to be diabetic [16]. If true, because of a high mortality rate among those with diabetes, the $\mathrm{Val} / \mathrm{Val}$ genotype should be associated with lower mortality rates. On the other hand, one might speculatively assume that diabetic individuals with Val/Val genotype have a less favorable prognosis and higher mortality than diabetics with other genotypes. Thus, how the Val158Met polymorphism influences on mortality among persons with diabetes mellitus is difficult to predict, but our exclusion of the 69 persons with self-reported diabetes probably minimize the risk of bias. However, the main results were not sub- 
stantially changed when the analyses were repeated including these 69 diabetic persons.

Our sample of 2979 individuals should have enough power to detect a difference in survival between genotypes that is of clinical interest, even when considering smaller subgroups deceased because of neoplasms or heart diseases. However, the number of individuals who died from accidents, suicide, prostate cancer, non-Hodgkin lymphoma, breast cancer or cancer in female genital organs was very low. For such relatively rare causes of death the impact of Val158Met polymorphism needs to be evaluated with other study designs. An example of this is a prospective study from United Kingdom of 2430 women with breast cancer reporting no effect on survival from polymorphisms in the COMT gene [20].

In the present study no association between overall cardiovascular mortality and Val158Met polymorphism was found. However, when the Met/Met and Val/Met genotypes were pooled, individuals with $\mathrm{Val} / \mathrm{Val}$ genotype tended to be less likely to die because of heart diseases classified as I30-I33 or I39-I52 compared to those with other genotypes, and the difference was significant even when we controlled for the most important confounding factors. It should be noted that this result may be due to chance because of the multiple hypotheses being tested. Hence, the association between the Val/Val genotype and death from non-ischemic heart disease should be confirmed in a lager study.

$\mathrm{Val} /$ Val genotype is associated with high COMT enzyme activity, and speculatively, rapid metabolism of circulating catecholamines may be protective for some type of heart diseases e.g. the arrhythmias. Theoretically, haplotypes of the COMT gene may influence COMT activity. However, because of lack of financial and personnel resources we did not evaluated other single nucleotide polymorphisms within the COMT gene or in other genes, and as a consequence, we did not have the opportunity to evaluate haplotypes of the COMT gene or possible interaction with other genes.

Few other studies have evaluated the influence of COMT genotypes on heart diseases. In accordance with our study, in a Finnish cohort of 773 men, Met/Met genotype and heavy coffee intake was associated with higher incidence of acute coronary events than those with other genotypes [16]. In contrast to our findings, a Swedish study found the Met/Met genotype to be associated with decreased risk of myocardial infarction, but this study was performed in a highly selected group of hypertensive individuals [12].

\section{Conclusion}

In this population-based study evaluating the impact of the COMT Val158Met polymorphism on survival among 2,979 adults, the polymorphism had no influence on total mortality.

\section{Methods Study population}

Between August 1995 and June 1997, all inhabitants aged 20 years or older in Nord-Trøndelag county in Norway ( $\mathrm{n}$ $=92,936)$ were invited to participate in the Nord-Trøndelag Health Survey ("Helseundersøkelsen i Nord-Trøndelag" = HUNT). In brief, two questionnaires including more than 200 health-related questions were administrated to the participants. The population in Nord-Trøndelag County was ethnically homogeneous (less than 3 percent of subjects were of non-white ethnicity), making it suitable for epidemiological genetic research [14].

Out of the 92,936 invited individuals, a total of 65,291 subjects (70 percent) answered the first questionnaire and participated in the health examination. Details of the non-participants are described elsewhere [14,15].

In the HUNT 2 biobank a total of 60,241 DNA samples were available. Genotyping of the COMT locus was performed among 3048 individuals. Approximately 70 percent were selected completely at random, whereas the remaining 30 percent included had been randomly selected among an older group of individuals who did not have self-reported diabetes mellitus. This latter group was generated in connection with a planned genetic study on diabetes that needed age-matched controls to a diabetic population. In the present sample the prevalence of selfreported diabetes mellitus was slightly lower among individuals with $\mathrm{Val} / \mathrm{Val}$ genotype than the other genotypes (2.0\% versus $2.3 \%, \mathrm{p}=0.61)$, and also in a Finnish study individuals with $\mathrm{Val} / \mathrm{Val}$ were less likely to be diabetic [16]. In the unidentified data file all participants were mixed, and separate analyses of the group selected completely by random was not possible. Thus, with intention to have a homogeneous study cohort, those with selfreported diabetes $(n=69)$ in the randomly selected group were eliminated [13], resulting in a population of 2979 individuals for the final analysis. All age groups were included, but because the age-matched controls to a diabetic population as a group are older than the general population, the total group of 2979 individuals without self-reported diabetes was 3.5 years older (mean 52.6 years versus 49.1 years) than the HUNT population as a whole.

\section{Causes of death}

We performed mortality follow-up by record linkage using the Norwegian 11-digit birth number (date of birth 
plus a 5-digit identifier), which is unique to each person residing in Norway, to obtain the date and underlying cause of death kept by Statistics Norway. The HUNT data including genotyping at the COMT locus were linked to the National Cause of Death register, and our report is based on mortality follow-up through the year 2004. We classified the 395 deaths on the basis of the underlying cause of death coded at Statistics Norway by using the 9th (death before 1996), or 10th revisions of the International Classification of Diseases (ICD). To further explore the influence of the Val158Met polymorphism on mortality from cancer and heart diseases on death, we also evaluated the immediate cause of death and up to five contributing causes of death. The underlying cause of death was defined as: a) the disease or injury that initiated the chain of morbid events leading directly to death or b) the external circumstances of the accident or violence that was the cause of the fatal injury [17]. By immediate cause of death was meant the disease, injury or condition directly leading to death and which was caused by the underlying cause of death [17]. Contributing cause of death was meant the condition that may have contributed to the death, but was not the direct causal relation with the disease or condition that has caused the death [17].

\section{Genotyping of the COMT locus}

Blood sampling was done whenever subjects attended, and details for the procedure and the HUNT 2 biobank are described elsewhere [14]. DNA for genotyping was extracted from peripheral blood leukocytes from whole blood or blood clots stored in the HUNT 2 biobank, using the Puregene kit (Gentra Systems Inc.) manually or with an Autopure LS (Gentra Systems Inc.). Laboratory technicians were blinded to the data from the National Cause of Death register. COMT genotypes were determined using the LightCycler (Roche Diagnostics Scandinavia AB, Bromma, Sweden) fluorescence resonance energy transfer method [18]. Polymerase chain reaction (PCR) amplifications were performed in $20 \mu \mathrm{l}$ reactions on a LightCycler System, using $2 \mu \mathrm{l}$ genomic DNA and the LightCyclerFastStart DNA Master Hybridization Probes kit (Roche Diagnostics Scandinavia AB, Bromma, Sweden). PCR primers (Eurogentec, Seraing, Belgium) and fluorescence labeled probes (PROLIGO, Paris, France) are shown in Table 1. Based on melting curve profiles, participants were classified as having Val/Val, Val/Met, or Met/Met genotypes. Details on PCR and melting curve conditions are available on request.

\section{Ethics}

The study was approved by the Regional Committee for Ethics in Medical Research, by the Norwegian Data Inspectorate, and by the Directorate for Health and Social Affairs.

\section{Statistical analysis}

Differences between continuous variables were tested with analyses of variance (one-way ANOVA) and between dichotomous variables with the chi-square test (including Fisher's exact test). Analyses used two-tailed estimation of significance, and $\mathrm{p}<0.05$ was considered to be statistically significant. A Kaplan-Meier method was performed to estimate the cumulative survival and differences between genotypes were tested with a log rank test. We used the Cox proportional hazards model to adjust for age, gender and other potential confounding factors, including smoking status, level of education, use of alcohol, blood pressure, coffee intake and body mass index.

Overall, our sample of 2979 individuals had more than 80 percent power to detect a 3 percent difference in total mortality and mortality by cancer or heart diseases between pooled genotypes with 95 percent certainty.

Statistical analyses were performed using the Statistical Package for the Social Sciences (SPSS), version 14.0 (SPSS Inc, Chicago).

\section{Abbreviations \\ COMT $=$ Catechol-O-methyltransferase}

HUNT = "Helseundersøkelsen i Nord-Trøndelag" (The Nord-Trøndelag Health Study)

Val = Valine

Met $=$ Methionine

ICD = International Classification of Diseases

\section{Competing interests}

The author(s) declare that they have no competing interests.

\section{Authors' contributions}

$\mathrm{KH}$ conceived of the study and performed the statistical analysis. KH, LJS, FS, and JAZ all participated in the design and drafted the manuscript. EP carried out the genotyping. All authors read and approved the final manuscript.

\section{Acknowledgements}

The Nord-Trøndelag Health Study (The HUNT study) is a collaboration between The HUNT Research Centre, Faculty of Medicine, The Norwegian University of Science and Technology (NTNU); Norwegian Institute of Public Health; and the Nord-Trøndelag County Council.

\section{References}

I. Lotta T, Vidgren J, Tilgmann C, Ulmanen I, Melén K, Julkunen I, Taskinen J: Kinetics of human soluble and membrane-bound Catechol O-Methyltransferase: A revised mechanism and description of the thermolabile variant of the enzyme. Biochemistry 1995, 34:4202-4210. 
2. Azzam A, Mathews CA: Meta-analysis of the association between the Catechol-O-methyltransferase gene and obsessive-compulsive disorders. Am J Med Genet 2003, I 23:64-69.

3. Woo JM, Yoon KS, Yu BH: Catechol-O-methyltransferase genetic polymorphism in panic disorder. Am J Psychiatry 2002, I 59: | 785-1787.

4. Zubieta JK, Heitzeg MM, Smith YR, Bueller JA, Xu K, Xu Y, Koeppe RA, Stohler CS, Goldman D: COMT val ${ }^{158}$ met Genotype affects $\mu$-opioid neurotransmitter responses to a pain stressor. Science 2003, 299: 1240-1243.

5. Skibola CF, Bracci PM, Paynter RA, Forrest MS, Agana L, Woodage T, Guegler K, Smith MT, Holly EA: Polymorhisms and haplotypes in the cytochrome P450 I A I, prolactin, and Catehol-O-methyltransferase genes and non-Hodgkin lymphoma risk. Cancer Epidemiol Biomarkers Prev 2005, |4:239|-240I.

6. Lavigne JA, Helzlsouer KJ, Huang HY, Strickland PT, Bell DA, Selmin O, Watson MA, Hoffman S, Comstock GW, Yager JD: An association between the allele coding for a low activity variant of catechol-O-methylytransferase activity and the risk for breast cancer. Cancer Res 1997, 57:5493-5497.

7. Goodman MT, McDuffie K, Kolonel LN, Terada K, Donlon TA, Wilkens LR, Guo C, Le Marchand L: Case-control study of ovarian cancer and polymorhisms in genes involved in catecholestrogen formation and metabolism. Cancer Epidemiol Biomarker Prev 2001, 10:209-216.

8. Suzuki M, Mamun MRI, Hara K, Ozeki T, Yamada Y, Kadowaki T, Honda H, Yanagihara Y, Ito YM, Kameyama S, Ohta N, Hosoi T, Arai T, Sawabe M, Takeuchi T, Takahashi S, Kitamura T: The Vall 58Met plomorhism of the Catechol-O-methyltransferase gene is associated with the PSA-progression-free survival in prostate cancer patient treated with estramustine phosphate. Eur Urol 2005, 48:752-759.

9. Ono H, Shirakawa O, Nushida H, Ueno Y, Maeda K: Association between Catechol-O-methyltransferase functional polymorphism and male suicide completers. Neuropsychopharm 2004, 29:1374-I377.

10. Lachman HM, Nolan KA, Mohr P, Saito T, Volavka J: Association between catechol-O-methyltransferase genotype and violence in schizophrenia and schizoaffective disorders. Am J Psychiatry 1998, I55;:835-837.

I I. Nolan KA, Volavka J, Czobor P, Cseh A, Lachman H, Saito T, Tiihonen J, Putkonen A, Hallikainen T, Kotilainen I, Rasanen P, Isohanni M, Jarvelin MR, Karvonen MK: Suicidal behavior in patients with schizophrenia is related to COMT polymorphism. Psychiatr Genet 2000, 10:117-124.

12. Eriksson AL, Skrtic S, Niklason A, Hultén LM, Wiklund O, Hedner T, Ohlsson C: Association between the low activity genotype of catechol-O-methyltransferase and myocardial infarction in a hypertensive population. Eur Heart J 2004, 25:386-39I

13. Hagen K, Stovner LJ, Pettersen E, Skorpen F, Holmen J, Zwart JA High systolic blood pressure is associated with $\mathrm{Val} / \mathrm{Val}$ genotype in the Catechol-O-methyltransferase gene - the HUNT Study. Am J Hypertens 20:21-26.

14. Holmen J, Midthjell K, Krüger $\varnothing$, Langhammer A, Holmen TL, Bratberg GH, Vatten L, Lund-Larsen PG: The Nord-Trøndelag Health Study 1995-97 (HUNT 2): Objectives, contents, methods and participation. Nor J Epidemiol 2003, I3:19-32.

15. Langhammer A, Johnsen R, Holmen J, Gulsvik A, Bjermer L: Cigarette smoking gives more respiratory symptoms among women than among men. In J Epidemiol Community Health Volume 54. The Nord-Trøndelag Health Study (HUNT); 2000:917-922

16. Happonen P, Voutilainen S, Tuomainen TP, Salonen JT: Catechol-omethyltransferase gene polymorphism modifies the effect of coffee intake on incidence of acute coronary events. PLOS ON 2006, I:el I7.

17. Statistics Norway, Causes of Death I99|-2000 2003:16-17 [http://www.ssb.no/dodsarsak].

18. Wittwer CT, Ririe KM, Andrew RV, David DA, Gundry RA, Balis UJ: The LightCycler: a microvolume multisample fluorimeter with rapid temperature control. Biotechniques 1997, 22: I76-|8I.

19. Manolio TA, Bailey-Wilson JE, Collins FS: Genes, environment and the value of prospective cohort studies. Nat Rev Genet 2006, 7:812-820.

20. Goode EL, Dunning AM, Kuschel B, Healey CS, Day NE, Ponder BAJ, Easton DF, Pharoah PPD: Effect of germ-line genetic variation on breast cancer survival in a population-based study. Cancer Research 2002, 62:3052-3057.

\section{Pre-publication history}

The pre-publication history for this paper can be accessed here:

http://www.biomedcentral.com/1471-2350/8/34/prepub
Publish with Bio Med Central and every scientist can read your work free of charge

"BioMed Central will be the most significant development for disseminating the results of biomedical research in our lifetime. "

Sir Paul Nurse, Cancer Research UK

Your research papers will be:

- available free of charge to the entire biomedical community

- peer reviewed and published immediately upon acceptance

- cited in PubMed and archived on PubMed Central

- yours - you keep the copyright 